\title{
RESEARCH HIGHLIGHT OPEN Microbiome harbored within tumors: a new chance to revisit our understanding of cancer pathogenesis and treatment
}

Kunming Zhao ${ }^{1,2}$ and Ying $\mathrm{Hu}^{1,2}$

Signal Transduction and Targeted Therapy (2020)5:136
; https://doi.org/10.1038/s41392-020-00244-1

\begin{abstract}
In a recent study in Science, Nejmen et al. provide the first comprehensive characterization of the tumor microbiome. Their data reveal that the microbiome mostly resides intracellularly in both tumor and immune cells, and that its composition varies with tumor type and subtype, smoking status, and immunotherapy response.
\end{abstract}

Mounting evidence suggests the active involvement of the tumor microenvironment in pathogenesis and treatment responses, ${ }^{1}$ leading to the newly accepted concept that tumors are more like holistic intricate biosystems than a collection of malignant cancer cells. ${ }^{2}$ Innate and adaptive immune cells, blood and lymphatic vessel networks, and stromal cells are general components that constitute the tumor microenvironment. Recently, an increasing number of tumors have been reported to harbor bacteria, raising the possibility that the microbiome may be an additional player in the complex tumor ecosystem. Nonetheless, due to their relatively low biomass and the suspicion of sample contamination, both the existence and function of the tumor microbiome remain issues under debate. In a recent publication in Science, Nejmen et al. ${ }^{1}$ accomplished, for the first time, a comprehensive analysis of the tumor microbiome, examining 1526 samples and corresponding normal adjacent tissue samples across seven types of tumors, including both common tumor types (breast, lung, ovary, and pancreatic) and others with little known microbiome information (melanoma, bone, and brain) using a single platform. To reduce the potential effects of contamination before and during analysis, the authors set up 437 DNA extraction controls, 206 PCR template-free controls, and 168 paraffin controls. In addition, they combined an improved $16 \mathrm{~S}$ ribosomal RNA gene-sequencing method with microscopy and culture-based techniques to characterize tumorresiding bacteria in a more precise manner. By doing so, they found that each tumor type had a distinct microbiome composition, despite a varied bacteria detection rate ranging from $14.3 \%$ in melanoma to $>60 \%$ in breast, pancreatic, and bone tumors. Remarkably, bacteria were found to be predominately intracellular and to reside in both tumor and immune cells. They further validated the associations between specific bacteria, tumor types and subtypes, smoking history, and sensitivity to immunotherapy, highlighting a potential function of the microbiome in tumor behavior and treatment responses. These important findings raise diverse issues that warrant further investigation (Fig. 1).

First, the fact that microbiomes are commonly harbored within tumors leads to an interesting question about what makes up the tumor microbiome. With advances in molecular and culture techniques, many tissues that have been traditionally considered sterile have been shown to harbor low-abundance microbial communities. The data from Nejmen et al. ${ }^{1}$ also reveal that the microbial compositions of different tumors are similar to those of their normal adjacent tissues. Thus, it is possible that tumor microbiomes evolve from bacteria that exist in normal tissues. Alternatively, the occurrence of tumor microbiomes may result from the systemic chronic inflammation, local vascular permeability, and immunosuppressive or metabolic status within tumors. ${ }^{3}$ Furthermore, in some types of tumors (i.e. pancreatic), the microbiome may be initiated by bacterial translocation from the intestinal tract. This model is supported by the observation that both pancreatic cancer and colon tissues harbor similar bacterial taxa. ${ }^{1}$ These bacteria may then be subjected to further selection by the distinctive pathological characteristics of the tumor, resulting in a relatively high bacterial load and temporally stable tumor-specific microbiome features.

Second, despite a significant association between the tumor microbiome and some pathological features of tumors, direct evidence regarding the effect of the tumor microbiome on the development of cancer is lacking. Microorganisms have been suspected to play a role in carcinogenesis for a long time. For example, the bacterium Helicobacter pylori has been classified as a human carcinogen that induces chronic inflammation in human gastric mucosa and gradually promotes the development of stomach cancer. Although preclinical and clinical studies suggest that, in most cases, microbially driven carcinogenesis is caused by a global change to the microbiome and not of a single pathogen, it is plausible that specific bacteria or microbiome compositions can influence the natural history of a tumor by modulating the host's immune response. In line with this idea, Riquelme and colleagues have successfully modulated the pancreatic tumor microbiome, which subsequently elicited a robust effect on tumor growth and tumor immune infiltration. ${ }^{4}$ In addition, emerging evidence has also shown an active role of bacteria in the development or progression and metastasis of malignancies by inducing chronic inflammation, genotoxicity, angiogenesis, or epithelial barrier failure. Whether tumor type-specific bacterial species or the microbiome features reported by Nejmen et al. ${ }^{1}$ contribute to the induction of the indicated tumors is not clear. Further, tumoral bacteria have the ability to shape the tumor immune microenvironment, which can influence the efficacy of immunotherapy, and have also been suggested to strongly interact with drugs, influencing drug sensitivity. ${ }^{5}$ Nejmen et al. ${ }^{1}$ show that microbiome features are associated with the immune

\footnotetext{
${ }^{1}$ Shenzhen Graduate School of Harbin Institute of Technology, Shenzhen 518055, China and ${ }^{2}$ School of Life Science and Technology, Harbin Institute of Technology, Harbin $150001 \mathrm{HL}$, China

Correspondence: Ying Hu (huying@hit.edu.cn)
}

Received: 10 June 2020 Accepted: 23 June 2020

Published online: 29 July 2020 


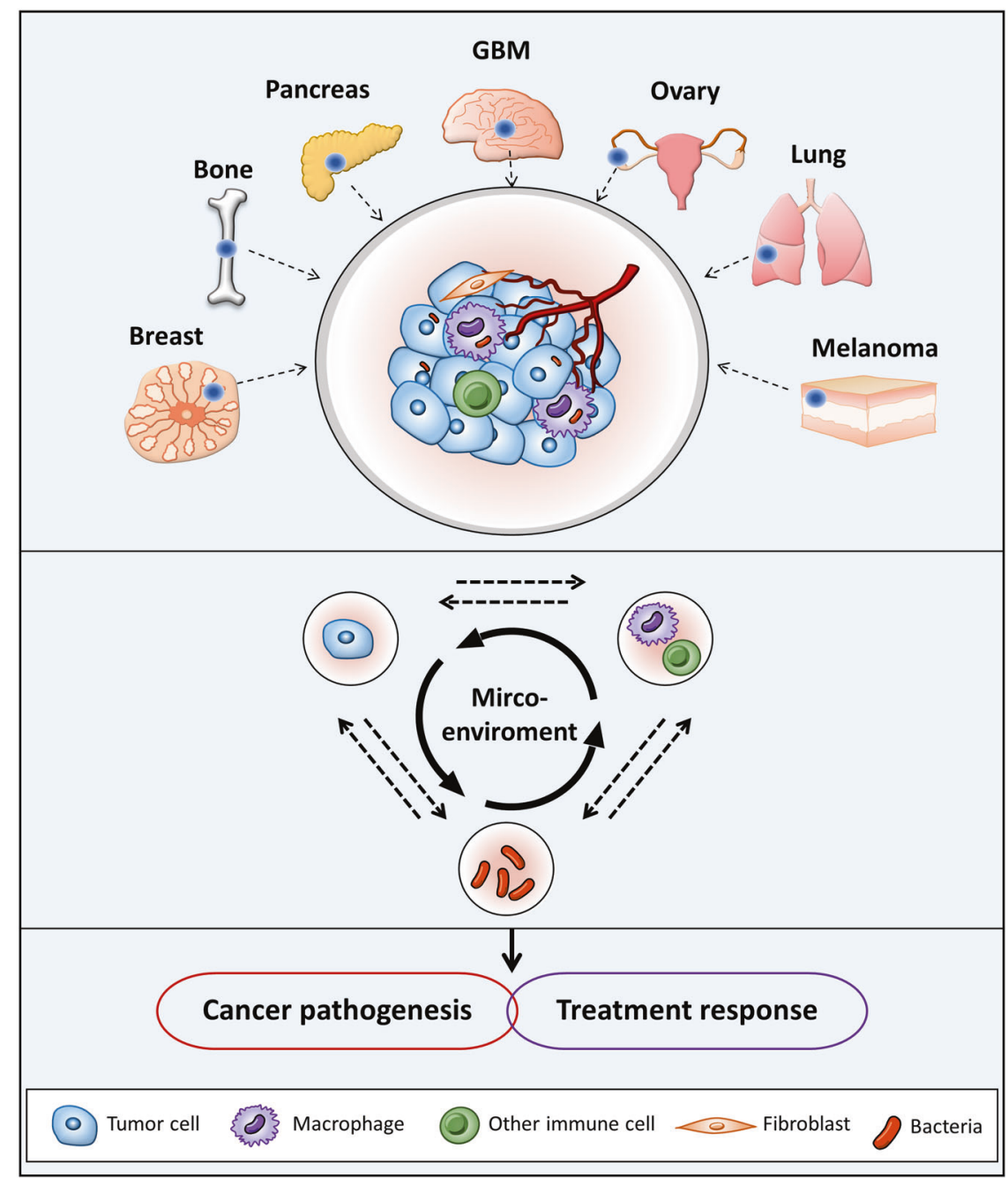

Fig. 1 Microbiome in tumors may be actively involved in microenvironment reconstruction and then play roles in the cancer pathogenesis and treatment response

response in melanoma, while the correlation between the microbiome and the efficacy of cytotoxic drugs or targeted therapies needs further exploration. It is possible that treatment efficacy may be improved by manipulating the tumor microbiome.

Third, if the tumor microbiome influences tumor behavior and treatment responses, further dissection of how the intricate web of tumor cells, the microbiome, immune cells, and other possible environmental components cause this is needed at the cellular and molecular level. An unexpected finding by Nejmen et al. ${ }^{1}$ is that bacteria reside mainly in the cytoplasms of tumor and immune cells. Intriguingly, such bacteria are metabolically active, so it is possible that intracellular bacteria influence biological processes, particularly those related to cancer hallmarks, via their autonomous metabolism. Alternatively, does the microbiome shape the tumor microenvironment via a non-cell-autonomous mechanism? If so, what molecules mediate such effects? Answers to these questions may facilitate the development of microbiometargeted treatments and improve the efficacies of current cancer treatments.

In summary, the comprehensive analysis of the tumor microbiome by Nejmen et al. ${ }^{1}$ is an essential first step toward deeper understanding of the influence of the tumor microbiome on the behaviors and responses of tumors to systemic therapies, which are a field of new opportunity in cancer biology. Future clinical studies considering these aspects are warranted and may help to improve the clinical efficacies of therapies.

\section{ACKNOWLEDGEMENTS}

This work was funded by the National Nature Science Foundation of China (No. 31301131, 31741084 and 31871389) and Basic Science Fundation of Science and technology innovation Commission in Shenzhen (No. JCYJ20170811154452255).

\section{ADDITIONAL INFORMATION}

Competing interests: The authors declare no competing interests.

\section{REFERENCES}

1. Nejmen, D. et al. The human tumor microbiome is composed of tumor type-specific intracellular bacteria. Science 368, 973-980 (2020).

2. Junttila, M. R. et al. Influence of tumour micro-environment heterogeneity on therapeutic response. Nature 501, 346-354 (2013).

3. Łaniewski, P. et al. The microbiome and gynaecological cancer development, prevention and therapy. Nat. Rev. Urol. 17, 232-250 (2020).

4. Riquelme, E. et al. Tumor microbiome diversity and composition influence pancreatic cancer outcomes. Cell 178, 795-806 (2019).

5. Gopalakrishnan, V. et al. The influence of the gut microbiome on cancer, immunity, and cancer immunotherapy. Cancer Cell. 33, 570-580 (2018). 
Microbiome harbored within tumors: a new chance to revisit our...

Zhao and $\mathrm{Hu}$

Open Access This article is licensed under a Creative Commons Attribution 4.0 International License, which permits use, sharing, adaptation, distribution and reproduction in any medium or format, as long as you give appropriate credit to the original author(s) and the source, provide a link to the Creative Commons license, and indicate if changes were made. The images or other third party material in this article are included in the article's Creative Commons license, unless indicated otherwise in a credit line to the material. If material is not included in the

(c) (i) article's Creative Commons license and your intended use is not permitted by statutory regulation or exceeds the permitted use, you will need to obtain permission directly from the copyright holder. To view a copy of this license, visit http://creativecommons. org/licenses/by/4.0/.

(c) The Author(s) 2020 